\title{
ESTUDIO PALINOLÓGICO DEL GÉNERO MACROPTILIUM (BENTH.) URB. (LEGUMINOSAE) EN MÉXICO Y CENTROAMÉRICA
}

\author{
Leticia Torres-Colín ${ }^{1}$, SARa Fuentes-Soriano $^{2}$ y Alfonso Delgado-Salinas ${ }^{1}$ \\ ${ }^{1}$ Universidad Nacional Autónoma de México, Instituto de Biología, Departamento \\ de Botánica. Apdo. postal 70-367, 04510 México D.F., México. \\ ${ }^{2}$ Missouri Botanical Garden, PO BOX 299, St. Louis MO 63116-0299, U.S.A. \\ responsable: lety@ibiologia.unam.mx
}

\section{RESUMEN}

Se describe la morfología del polen de ocho especies del género Macroptilium con distribución en México y Centroamérica, a partir de granos acetolizados y observados bajo microscopio de luz y microscopio electrónico de barrido. En esta región geográfica los miembros del género tienen granos de polen tricolporados, prolado-esferoidales, suboblados a rara vez oblado-esferoidales, de tamaño generalmente mediano. De acuerdo con las diferencias en las ectoaberturas (longitud, forma del ápice y membrana), ornamentación de la exina, engrosamiento del margo y contorno en vista polar se distinguen dos tipos polínicos: Tipo I. Polen con colpos cortos (brevicolpos), margo engrosado, ápice de la ectoabertura redondeado, membrana granular, contorno angular convexo, exina tectada o semitectada, punctada a rugulada en el apocolpio y microrreticulada a rugulada en el mesocolpio; característico de la sección Microcochle, representada por M. gibbosifolium, M. pedatum y M. supinum. Tipo II. Polen con colpos largos (longicolpos), margo no engrosado, ápice de la ectoabertura agudo, membrana granular, contorno circular a semiangular, exina tectada a semitectada, psilada, punctada, foveolada a microrreticulada en el apocolpio y microrreticulada a foveolada en el mesocolpio; este tipo distingue a las especies de la sección Macroptilium, incluyendo a M. atropurpureum, M. erythroloma, M. gracile, M. lathyroides y $M$. longipedunculatum. Por primera vez se proveen descripciones palinológicas para cinco especies del género.

Palabras clave: Centroamérica, Leguminosae, Macroptilium, México, polen.

\section{ABSTRACT}

The pollen morphology of eight Macroptilium species occurring in Mexico and Central America is described by means of light and scanning microscopy. In this region, 
members of the genus have pollen grains 3-colporate, medium-sized, with polar shapes from prolate-spheroidal, suboblate to oblate-spheroidal. Differences in ectoaperture (length, apex shape and membrane), ornamentation, margo, and the outline in polar side identify two pollen types: Type I. Pollen with small colpi (brevicolpate), margo thickened, apex of the ectoaperture round, membrane granular, polar outline convex angular, exine tectate or semitectate, apocolpium punctate or rugulate, and mesocolpium microreticulate or rugulate. Species of section Microcochle, including M. gibbosifolium, M. pedatum, and M. supinum are characterized by this pollen type. Type II. Pollen with long colpi (longicolpate) margo non-thickened, apex of the ectoaperture acute, membrane granular, pollen outline circular to semi-angular, exine tectate or semitectate, apocolpium punctate to foveolate, and mesocolpium microreticulate to foveolate. Species of section Macroptilium comprising, $M$. atropurpureum, M. erythroloma, M. gracile, M. lathyroides, and M. longipedunculatum are distinguished by this pollen type. Palynological descriptions of five species of Macroptilium are provided for the first time.

Key words: Central America, Leguminosae, Macroptilium, Mexico, pollen.

\section{INTRODUCCIÓN}

El género Macroptilium está representado por 18 especies (Drewes, 1997), distribuidas en los trópicos y subtrópicos del continente americano, desde el sur de los Estados Unidos, hasta el norte de la República de Argentina y el Uruguay. A partir de que Macroptilium fue elevado a rango genérico por Urban en 1928, se han publicado más de una docena de nuevas combinaciones y sólo una nueva especie (Maréchal, 1974; Maréchal \& Baudet, 1977; Maréchal et al., 1978a; Juárez \& Pérez, 1986, 1987; Drewes \& Palacios, 1994, 1995; Delgado-Salinas \& Torres-Colín, 1995, 2004; Delgado-Salinas \& Lewis, 2008), aunque se ha considerado en algunas revisiones regionales de Brasil (Fevereiro, 1987), Argentina (Drewes, 1997, 2001), Cuba (Beyra \& Reyes, 2005), México y Centroamérica (Torres-Colín, 2006), y recientemente en un estudio de datos morfológicos, bioquímicos y moleculares que sustentan la primera propuesta filogenética para el conjunto (Espert et al., 2007).

En la revisión taxonómica del género, basada en una exhaustiva revisión morfológica de caracteres vegetativos y florales, Torres-Colín (2006) distinguió claramente dos secciones. En este estudio la sección Microcochle incluyó a Macroptilium gibbosifolium, M. pedatum, y a M. supinum, todas ellas caracterizadas con raíces globosas, suculentas, hábito postrado, estípulas extendidas, nectarios extraflorales en los nudos de la inflorescencia no hinchados, brácteas pedunculares caducas, ovario rodeado en su base por un cinturón nectarífero tubular; valvas de los frutos con torsión al hacer dehiscencia, semillas con la testa rugulada a simple-foveolada e hilo 
ovado y todas desarrollan inflorescencias con flores cleistogámicas. La sección $M a-$ croptilium incluyó a $M$. atropurpureum, M. erythroloma, M. gracile, M. lathyroides y M. longipedunculatum con raíces pivotantes, alargadas, hábito trepador o erecto, estípulas retrorsas, nectarios extraflorales hinchados en los nudos de la inflorescencia, brácteas pedunculares generalmente persistentes, ovario rodeado en su base por un cinturón nectarífero tubular sesgado, valvas de los frutos sin torsión al hacer dehiscencia, semillas con la testa simple-reticulada a multireticulada e hilo oblongo y sólo una especie con desarrollo de cleistogamia (M. longipedunculatum).

El estudio de polen en Macroptilium ha estado limitado a algunas descripciones aisladas, basadas en su mayoría en observaciones realizadas bajo microscopio óptico y con interpretaciones hechas dentro del género Phaseolus L. (Taylor, 1966; Verdcourt, 1970; Melhem, 1971; Stainier, 1974). En investigaciones más recientes e incorporando técnicas de microscopio electrónico de barrido, se observaron detalles en la morfología del polen que permitieron circunscribir los géneros en complejos cercanos a Macroptilium (Maréchal et al., 1978b) y delimitar secciones y especies en este último (Drewes, 1996).

Los objetivos del presente estudio son analizar en detalle las variaciones palinológicas del género en México y Centroamérica, proveer descripciones palinológicas completas y comparables para los componentes de Macroptilium en esta región y evaluar la utilidad taxonómica de caracteres palinológicos en la delimitación de sus especies.

\section{MATERIALES Y MÉTODOS}

Se analizaron 38 muestras obtenidas de ejemplares del herbario (MEXU), correspondientes a ocho especies de Macroptilium (ver Apéndice), las que fueron acetolizadas siguiendo el método de Erdtman (1960), con una extensión en los tiempos de acetólisis (aproximadamente por 12 minutos); dicha modificación no alteró el aspecto general de los granos de polen, únicamente ayudó a visualizar las diferencias encontradas en el tamaño de las aberturas (Torres-Colín, 2006).

Para las observaciones en microscopio óptico de marca Carl Zeiss Standard (MO), una parte de la mezcla se montó en gelatina glicerinada y se hicieron de 3 a 5 preparaciones permanentes; las medidas se basaron en 60 granos de polen en vista ecuatorial y 60 en vista polar con más de tres repeticiones por especie (excepto para M. pedatum, taxon endémico a México, para el cual se contó con poco material). Las microfotografías se tomaron en el mismo microscopio equipado con una cámara 
adaptada, modelo MC80, bajo campo claro en el objetivo de $100 \mathrm{X}$ y se usó película Ilford Pan F-Plus, ISO 50 en blanco y negro. Las preparaciones se encuentran depositadas en la palinoteca del Instituto de Biología, de la Universidad Nacional Autónoma de México. La otra parte de las muestras fue deshidratada en alcoholes graduales de $50 \%$ a $100 \%$ por 10 minutos y éstas posteriormente se secaron con la técnica de secado a punto crítico. Se cubrieron con una capa de oro-paladio para ser observadas y fotografiadas bajo un microscopio electrónico de barrido (MEB) marca Hitachi modelo S2460-N, de igual forma las imágenes se registraron en película Ilford Pan F-Plus, ISO 50 en blanco y negro.

La terminología que se siguió para describir la morfología del polen y la ornamentación de la exina fue la de Erdtman (1952), Praglowski y Punt (1973), Saxena (1993) y Punt et al. (1994).

\section{RESULTADOS}

Los granos de polen de Macroptilium son tricolporados, prolado-esferoidales, oblado-esferoidales a rara vez suboblados y de tamaño medio. Presentan ectoaberturas en brevicolpos de 11-13.3 $\mu \mathrm{m}$ de largo o longicolpos de 20.7-29.5 $\mu \mathrm{m}$ de largo, con un ápice agudo a redondeado y una membrana granular, bordeadas por un margo engrosado o no engrosado que varía de psilado, punctado a rugulado. Las endoaberturas son lolongadas o lalongadas. En vista polar el contorno es angular convexo, semiangular o circular, con una área polar grande a media. La exina mide de 1.3-2.8 $\mu \mathrm{m}$ de grosor, es tectada o semitectada y varía de psilada, punctada, foveolada, microrreticulada a rugulada en el apocolpio y de microrreticulada, foveolada a rugulada en el mesocolpio.

Se reconocieron dos tipos polínicos, con base en longitud, ápices del colpo, margo, ornamentación de la exina (con variación en apocolpio y mesocolpio) y contorno en vista polar.

Descripción de los tipos polínicos y del polen de las especies correspondientes

Tipo I. Incluye especies con granos tricolporados, oblado-esferoidales, prolado esferoidales a suboblados, de 26.4-37.8 $\mu \mathrm{m}$ de longitud polar y 28.0-40.9 de longitud ecuatorial. Ectoaberturas en brevicolpos (colpos de menos de $15 \mu \mathrm{m}$ de longitud) con ápices redondeados, membranas granulares y margos engrosados (2.4$3.5 \mu \mathrm{m}$ de grosor) que varían de psilados, punctados a rugulados. Endoaberturas 
lolongadas. En vista polar el contorno es angular convexo, con un área polar grande. Exina tectada a semitectada, punctada a rugulada en el apocolpio y microrreticulada a rugulada en el mesocolpio con lúmenes con o sin gránulos.

Especies incluidas: M. gibbosifolium, M. pedatum y M. supinum (Cuadro 1).

1. Macroptilium gibbosifolium (Ortega) A. Delgado (Figs. 1-5F).

MO: Granos tricolporados de tamaño medio, oblado-esferoidales. Ectoabertura en brevicolpo de $13.3 \mu \mathrm{m}$ de longitud polar y de $3.6 \mu \mathrm{m}$ de longitud ecuatorial, ápice redondeado, membrana granular; margo engrosado con variación en la ornamentación, frecuentemente psilado a ocasionalmente punctado, de 2.4-3.5 $\mu \mathrm{m}$ de grosor. Endoabertura lolongada de $6.9 \mu \mathrm{m}$ de longitud polar y $4.3 \mu \mathrm{m}$ de longitud ecuatorial. Exina de $1.3 \mu \mathrm{m}$ de grosor, sexina ligeramente más delgada que la nexina. En vista polar el contorno es angular convexo, distancia entre colpos $22.6 \mu \mathrm{m}$. Área polar grande, con un índice de $0.6 \mu \mathrm{m}$.

MEB: Apocolpio tectado, punctado y mesocolpio semitectado, microrreticulado; lúmenes con o sin gránulos.

2. Macroptilium pedatum (Rose) Maréchal \& Baudet (Figs. 2G; 3-5H).

MO: Granos tricolporados de tamaño medio, prolado-esferoidales. Ectoabertura en brevicolpo cuyas dimensiones no se pudieron medir, ápice redondeado, membrana granular; margo engrosado con variación en la ornamentación, frecuentemente rugulado a ocasionalmente psilado, de $3.03 \mu \mathrm{m}$ de grosor. Endoabertura lolongada, de $5.6 \mu \mathrm{m}$ de longitud polar y de $4.2 \mu \mathrm{m}$ de longitud ecuatorial. Exina de $2.8 \mu \mathrm{m}$ de grosor, sexina más gruesa que la nexina. En vista polar el contorno es angular convexo, distancia entre colpos $21.4 \mu \mathrm{m}$. Área polar grande, con un índice de $0.5 \mu \mathrm{m}$.

MEB: Apocolpio y mesocolpio semitectados, rugulados; lúmenes sin gránulos.

3. Macroptilium supinum (Wiggins \& Rollins) A. Delgado \& L. Torres-Colín (Figs. $1,3,4,5 \mathrm{G} ; 2 \mathrm{H})$.

MO: Granos tricolporados de tamaño medio, suboblados. Ectoabertura en brevicolpo de $11.1 \mu \mathrm{m}$ de longitud polar y de $9.8 \mu \mathrm{m}$ de longitud ecuatorial, ápice 
redondeado, membrana granular; margo engrosado con variación en la ornamentación, frecuentemente punctado a ocasionalmente psilado, de $2.6 \mu \mathrm{m}$ de grosor. Endoabertura lolongada, de $7.1 \mu \mathrm{m}$ de longitud polar y de $5.9 \mu \mathrm{m}$ de longitud ecuatorial. Exina de $1.3 \mu \mathrm{m}$ de grosor, sexina ligeramente más delgada que la nexina. En vista polar el contorno es angular convexo, distancia entre colpos $23.2 \mu \mathrm{m}$. Área polar grande, con un índice de $0.6 \mu \mathrm{m}$.

MEB: Apocolpio tectado, punctado y mesocolpio semitectado, microrreticulado; lúmenes con o sin gránulos.

Tipo II. Incluye especies con granos tricolporados, prolado-esferoidales, de 31.6-46.6 $\mu \mathrm{m}$ de longitud polar y 29.9-45.7 $\mu \mathrm{m}$ de longitud ecuatorial. Ectoaberturas en longicolpos (colpos de 20.7 a $29.5 \mu \mathrm{m}$ de longitud) con ápices agudos, membranas granulares y margos no engrosados (1.0-2.8 $\mu \mathrm{m}$ de grosor) que varían de psilados, punctados a ligeramente microrrugulados. Endoaberturas lolongadas y lalongadas. En vista polar el contorno es circular o semiangular con un área polar generalmente de tamaño mediano. Exina tectada o semitectada, psilada, punctada, foveolada a micrroreticulada en el apocolpio y semitectada microrreticulada a foveolada en el mesocolpio con lúmenes con o sin gránulos.

Especies incluidas: M. atropurpureum, M. erythroloma, M. gracile, M. lathyroides y $M$. longipedunculatum (Cuadro 1).

4. Macroptilium atropurpureum (Sessé \& Moc. ex DC.) Urb. (Figs. 1-5A).

MO: Granos tricolporados de tamaño medio, prolado-esferoidales. Ectoabertura en longicolpo de $28 \mu \mathrm{m}$ de longitud polar y de $4.6 \mu \mathrm{m}$ de longitud ecuatorial, ápice agudo, membrana granular; margo no engrosado, con variación en la ornamentación, generalmente psilado a ocasionalmente punctado, de 1.5-2.8 $\mu \mathrm{m}$ de grosor. Endoabertura lolongada de $8.8 \mu \mathrm{m}$ de longitud polar y de $4.8 \mu \mathrm{m}$ de longitud ecuatorial. Exina de $2.2 \mu \mathrm{m}$ de grosor, sexina del mismo grosor que la nexina. En vista polar el contorno es semiangular, distancia entre colpos $17.7 \mu \mathrm{m}$. Área polar de tamaño medio, con un índice de $0.4 \mu \mathrm{m}$.

MEB: Apocolpio tectado, punctado a foveolado y mesocolpio semitectado, microrreticulado; lúmenes con más de un gránulo.

5. Macroptilium erythroloma (Mart. ex Benth.) Urb. (Figs. 1-5B). 
Cuadro 1. Detalles del polen de los dos tipos polínicos encontrados en Macroptilium. $\mathrm{MGI}=$ M. gibbosifolium; $\mathrm{MPE}=$ M. pedatum; MSU=M. supinum; MAT=M. atropurpureum; $\mathrm{MER}=M$. erythroloma $; \mathrm{MGR}=M$. gracile; $\mathrm{MLA}=$ M. lathyroides; $\mathrm{MLO}=M$. longipedunculatum; $\mathrm{E} . \mathrm{P} .=$ eje polar; $\mathrm{E} . \mathrm{E} .=\mathrm{eje}$ ecuatorial; $\mathrm{D} / \mathrm{P}=$ diámetro polar entre diámetro ecuatorial; $\mathrm{NM}=$ no medido.

\begin{tabular}{|c|c|c|c|c|c|c|c|c|c|c|c|c|}
\hline Especie & Forma & E.P. $\mu \mathrm{m}$ & E.E. $\mu \mathrm{m}$ & $\begin{array}{l}\mathrm{D} / \mathrm{P} \\
\mu \mathrm{m}\end{array}$ & $\begin{array}{l}\text { Contorno } \\
\text { polar }\end{array}$ & Aberturas & $\begin{array}{l}\text { Tipo de exina y } \\
\text { ornamentación } \\
\text { del apocolpio/ } \\
\text { mesocolpio }\end{array}$ & $\begin{array}{l}\text { Margo/ } \\
\text { ápice } \\
\text { ectoabertura }\end{array}$ & $\begin{array}{l}\text { Endoabertura } \\
\mu \mathrm{m}\end{array}$ & $\begin{array}{l}\text { Colpo } \\
\text { long/ancho } \\
\mu \mathrm{m}\end{array}$ & $\begin{array}{l}\text { Membrana } \\
\text { del colpo }\end{array}$ & $\begin{array}{l}\text { Exina } \\
\mu \mathrm{m}\end{array}$ \\
\hline $\begin{array}{l}\text { MGI } \\
\text { (Tipo I) }\end{array}$ & $\begin{array}{l}\text { oblado- } \\
\text { esferoidal }\end{array}$ & $26.4-32.1$ & $28.1-36.0$ & 0.97 & $\begin{array}{l}\text { angular } \\
\text { convexo }\end{array}$ & $\begin{array}{l}\text { tricolporado } \\
\text { brevicolpado }\end{array}$ & $\begin{array}{l}\text { tectada punctada } \\
\text { /semitectada } \\
\text { microrreticulada }\end{array}$ & $\begin{array}{l}\text { engrosado/ } \\
\text { redondeado }\end{array}$ & $6.9-4.3$ & $13.3 / 3.6$ & granular & 1.3 \\
\hline $\begin{array}{l}\text { MPE } \\
\text { (Tipo I) }\end{array}$ & $\begin{array}{l}\text { prolado- } \\
\text { esferoidal }\end{array}$ & $32.0-35.0$ & $28.0-34.0$ & 1.06 & $\begin{array}{l}\text { angular } \\
\text { convexo }\end{array}$ & $\begin{array}{l}\text { tricolporado } \\
\text { brevicolpado }\end{array}$ & $\begin{array}{l}\text { semitectada } \\
\text { rugulada/ } \\
\text { semitectada } \\
\text { rugulada }\end{array}$ & $\begin{array}{l}\text { engrosado/ } \\
\text { redondeado }\end{array}$ & $5.6-4.2$ & NM & granular & 2.8 \\
\hline $\begin{array}{l}\text { MSU } \\
\text { (Tipo I) }\end{array}$ & suboblado & 29.9-37.8 & $35.2-40.9$ & 0.83 & $\begin{array}{l}\text { angular } \\
\text { convexo }\end{array}$ & $\begin{array}{l}\text { tricolporado } \\
\text { brevicolpado }\end{array}$ & $\begin{array}{l}\text { tectada punctada/ } \\
\text { semitectada } \\
\text { microrreticulada }\end{array}$ & $\begin{array}{l}\text { engrosado/ } \\
\text { redondeado }\end{array}$ & $7.1-5.9$ & $11.1 / 9.8$ & granular & 1.3 \\
\hline $\begin{array}{l}\text { MAT } \\
\text { (Tipo II) }\end{array}$ & $\begin{array}{l}\text { prolado- } \\
\text { esferoidal }\end{array}$ & $36.0-40.9$ & $30.8-40.0$ & 1.1 & semiangular & $\begin{array}{l}\text { tricolporado } \\
\text { longicolpado }\end{array}$ & $\begin{array}{l}\text { tectada punctada } \\
\text { a foveolada / } \\
\text { semitectada } \\
\text { microrreticulada }\end{array}$ & $\begin{array}{l}\text { no } \\
\text { engrosado/ } \\
\text { agudo }\end{array}$ & $8.8-4.8$ & $28.0 / 4.6$ & granular & 2.2 \\
\hline $\begin{array}{l}\text { MER } \\
\text { (Tipo II) }\end{array}$ & $\begin{array}{l}\text { prolado- } \\
\text { esferoidal }\end{array}$ & $44.0-46.6$ & $38.7-45.7$ & 1.05 & circular & $\begin{array}{l}\text { tricolporado } \\
\text { longicolpado }\end{array}$ & $\begin{array}{l}\text { tectada psilada/ } \\
\text { semitectada } \\
\text { microrreticulada }\end{array}$ & $\begin{array}{l}\text { no } \\
\text { engrosado/ } \\
\text { agudo }\end{array}$ & $10.6-5.1$ & $29.5 / 4.8$ & granular & 1.7 \\
\hline $\begin{array}{l}\text { MGR } \\
\text { (Tipo II) }\end{array}$ & $\begin{array}{l}\text { prolado- } \\
\text { esferoidal }\end{array}$ & $31.6-37.8$ & $29.9-35.2$ & 1.07 & $\begin{array}{l}\text { circular a } \\
\text { semiangular }\end{array}$ & $\begin{array}{l}\text { tricolporado } \\
\text { longicolpado }\end{array}$ & $\begin{array}{l}\text { semitectada } \\
\text { microrreticulada/ } \\
\text { semitectada } \\
\text { microrreticulada }\end{array}$ & $\begin{array}{l}\text { no } \\
\text { engrosado/ } \\
\text { agudo }\end{array}$ & $7.4-6.6$ & $20.7 / 4.1$ & granular & 1.3 \\
\hline $\begin{array}{l}\text { MLA } \\
\text { (Tipo II) }\end{array}$ & $\begin{array}{l}\text { prolado- } \\
\text { esferoidal }\end{array}$ & $39.6-44.4$ & $35.2-42.2$ & 1.07 & $\begin{array}{c}\text { circular a } \\
\text { semiangular }\end{array}$ & $\begin{array}{l}\text { tricolporado } \\
\text { longicolpado }\end{array}$ & $\begin{array}{l}\text { tectada punctada } \\
\text { a foveolada/ } \\
\text { semitectada } \\
\text { microrreticulada a } \\
\text { foveolada }\end{array}$ & $\begin{array}{l}\text { no } \\
\text { engrosado/ } \\
\text { agudo }\end{array}$ & $8.7-7.5$ & $29.1 / 5.5$ & granular & 1.7 \\
\hline $\begin{array}{l}\text { MLO } \\
\text { (Tipo II) }\end{array}$ & $\begin{array}{l}\text { prolado- } \\
\text { esferoidal }\end{array}$ & $33.0-40.9$ & $30.8-37.8$ & 1.1 & $\begin{array}{c}\text { circular a } \\
\text { semiangular }\end{array}$ & $\begin{array}{l}\text { tricolporado } \\
\text { longicolpado }\end{array}$ & $\begin{array}{l}\text { tectada punctada } \\
\text { a foveolada / } \\
\text { semitectada } \\
\text { microrreticulada }\end{array}$ & $\begin{array}{l}\text { no } \\
\text { engrosado/ } \\
\text { agudo }\end{array}$ & $5.9-5.3$ & $24.6 / 3.9$ & granular & 2.2 \\
\hline
\end{tabular}


MO: Granos tricolporados de tamaño medio, prolado-esferoidales. Ectoabertura en longicolpo de $29.5 \mu \mathrm{m}$ de longitud polar y de $4.8 \mu \mathrm{m}$ de longitud ecuatorial, ápice agudo, membrana granular; margo no engrosado, con variación en la ornamentación, generalmente psilado, de $1.9 \mu \mathrm{m}$ de grosor. Endoabertura lolongada de $10.6 \mu \mathrm{m}$ de longitud polar y de $5.1 \mu \mathrm{m}$ de longitud ecuatorial. Exina de $1.7 \mu \mathrm{m}$ de grosor, sexina del mismo grosor que la nexina. En vista polar el contorno es circular, distancia entre colpos $30.7 \mu \mathrm{m}$. Área polar grande, con un índice de $0.6 \mu \mathrm{m}$.

MEB: Apocolpio tectado, psilado y mesocolpio semitectado, microrreticulado; lúmenes sin gránulos.

\section{Macroptilium gracile (Poepp. ex Benth.) Urb. (Figs. 1-5C).}

MO: Granos tricolporados de tamaño medio, prolado-esferoidales. Ectoabertura en longicolpo de $20.7 \mu \mathrm{m}$ de longitud polar y de $4.1 \mu \mathrm{m}$ de longitud ecuatorial, ápice agudo, membrana granular; margo no engrosado, con variación en la ornamentación, frecuentemente psilado a ocasionalmente punctado, de $2 \mu \mathrm{m}$ de grosor. Endoabertura lolongada, de $7.4 \mu \mathrm{m}$ de longitud polar y de $6.6 \mu \mathrm{m}$ de longitud ecuatorial. Exina de $1.3 \mu \mathrm{m}$ de grosor, sexina del mismo grosor que la nexina. En vista polar el contorno circular a semiangular, distancia entre colpos $15.6 \mu \mathrm{m}$. Área polar de tamaño medio, con un índice de $0.4 \mu \mathrm{m}$.

MEB: Apocolpio y mesocolpio semitectados, microrreticulados; lúmenes con más de un gránulo.

\section{Macroptilium lathyroides (L.) Urb. (Figs. 1-5D).}

MO: Granos tricolporados de tamaño medio, prolado-esferoidales. Ectoabertura en longicolpo de $29.1 \mu \mathrm{m}$ de longitud polar y de $5.5 \mu \mathrm{m}$ de longitud ecuatorial, ápice agudo, membrana granular; margo no engrosado, con variación en la ornamentación, generalmente psilado, de $1.4 \mu \mathrm{m}$ de grosor. Endoabertura lolongada de $8.7 \mu \mathrm{m}$ de longitud polar y de $7.5 \mu \mathrm{m}$ de longitud ecuatorial. Exina de $1.7 \mu \mathrm{m}$ de grosor, sexina del mismo grosor que la nexina. En vista polar el contorno es circular a semiangular, distancia entre colpos $14.8 \mu \mathrm{m}$. Área polar de tamaño medio, con un índice de $0.3 \mu \mathrm{m}$.

MEB: Apocolpio tectado, punctado a foveolado y mesocolpio semitectado microrreticulado a foveolado; lúmenes con un gránulo u ocasionalmente más de uno. 
8. Macroptilium longipedunculatum (Mart. ex Benth.) Urb. (Figs. 1-5E)

MO: Granos tricolporados de tamaño medio, prolado-esferoidales. Ectoabertura en longicolpo de $24.6 \mu \mathrm{m}$ de longitud polar y de $3.9 \mu \mathrm{m}$ de longitud ecuatorial, ápice agudo, membrana granular; margo no engrosado, con variación en la ornamentación, generalmente psilado a ocasionalmente microrrugulado o punctado, de 1.0-2.3 $\mu \mathrm{m}$ de grosor. Endoabertura lalongada, de $5.9 \mu \mathrm{m}$ de longitud polar y de 5.3 $\mu \mathrm{m}$ de longitud ecuatorial. Exina de $2.2 \mu \mathrm{m}$ de grosor, sexina más gruesa que la nexina. En vista polar el contorno es circular a semiangular, distancia entre colpos $14.1 \mu \mathrm{m}$. Área polar de tamaño medio, con un índice de $0.3 \mu \mathrm{m}$.

MEB: Apocolpio tectado, punctado a foveolado y mesocolpio semitectado, microrreticulado; lúmenes por lo general con más de un gránulo y ocasionalmente sin gránulos.

\section{DISCUSIÓN}

Aunque los granos de polen de Macroptilium son uniformes en tamaño y tipo de aberturas, diferencias en longitud de la ectoabertura, ápice del colpo, margo, ornamentación del polen y contorno en vista polar, permitieron distinguir dos tipos polínicos (Cuadro 1), que se correlacionan taxonómicamente con las dos secciones reconocidas por Torres-Colín (2006) y Espert et al. (2007).

El Tipo I está integrado por M. gibbosifolium, M. pedatum y M. supinum (sección Microcochle) y el Tipo II por M. atropurpureum, M. erythroloma, M. gracile, M. lathyroides y M. longipedunculatum (sección Macroptilium).

El tamaño encontrado no es un carácter taxonómico relevante en Macroptilium, cae en la categoría de granos de clase media de Erdtman (1952) en ambos tipos, con un intervalo de 26.4-46.6 $\mu \mathrm{m}$ (P) y 28.0-45.7 $\mu \mathrm{m}$ (E) (Cuadro 1), lo que confirma lo registrado por Taylor (1966) y Drewes (1996). En cuanto a la forma, ésta distingue claramente a las especies del Tipo I, es oblado-esferoidal en Macroptilium gibbosifolium (Fig. 1F), prolado-esferoidal en Macroptilium pedatum (Fig. 3H) y suboblada en Macroptilium supinum (Fig. 1G); en cambio en el Tipo II es prolado-esferoidal en todas las especies (Figs. 1A-E y 3A-E).

Las aberturas son del tipo tricolporado en ambos tipos, pero se encontraron variaciones en la longitud de la ectoabertura, que permitieron diferenciarlos. Aunque en algunos trabajos, como los de Melhem (1971), Stainier (1974) y Drewes (1996), se menciona la existencia de polen triporado para especies de Macroptilium, 

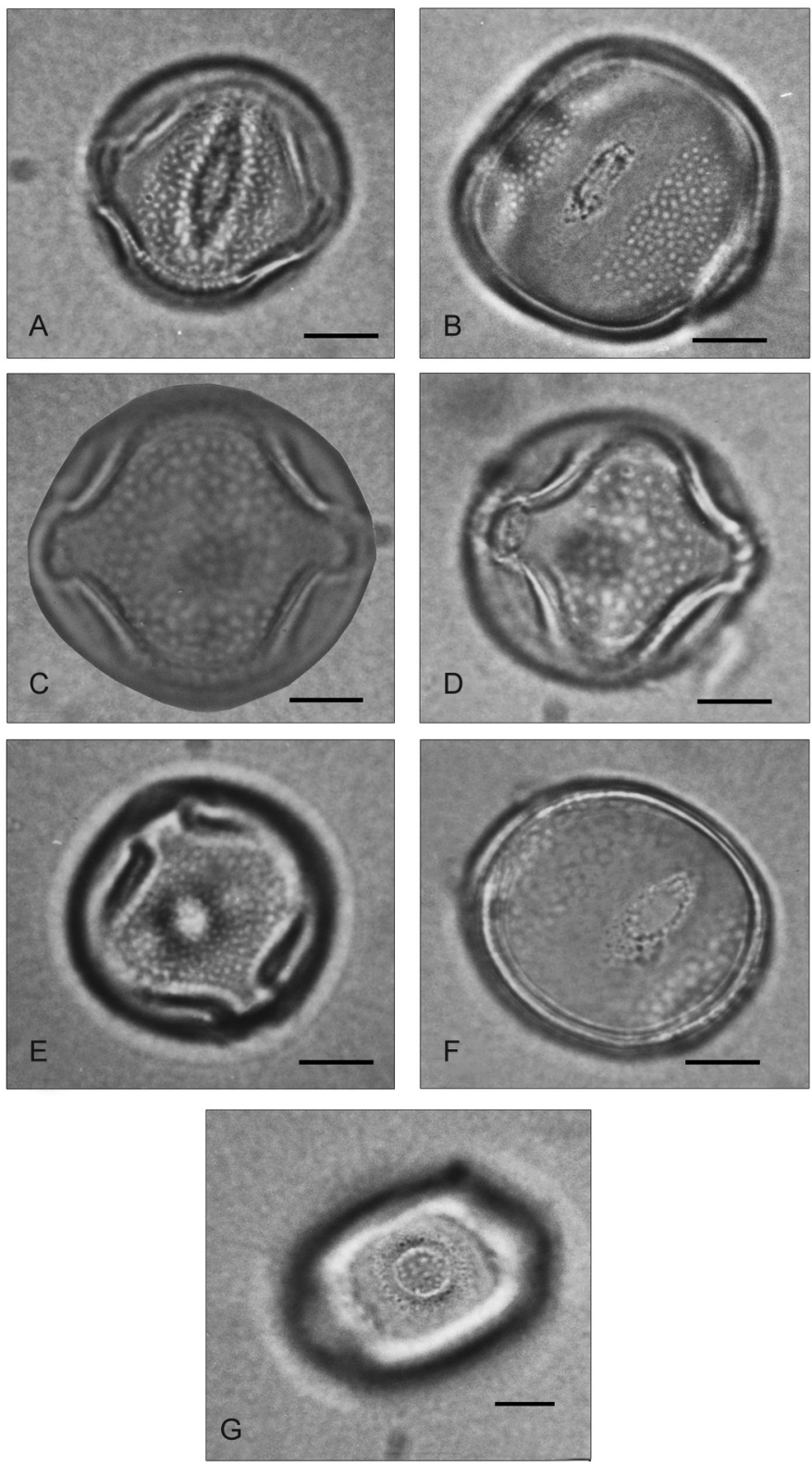

Fig. 1. Vista ecuatorial, granos de polen acetolizados de Macroptilium (microscopio óptico en campo claro). Se observa en B y F el margo y en A, B, F y G las aberturas. A. M. atropurpureum. B. M. erythroloma. C. M. gracile. D. M. lathyroides. E. M. longipedunculatum (sección Macroptilium). F. M. gibbosifolium. G. M. supinum (sección Microcochle). En A, B, D, E y $\mathrm{F}$ la escala $=4 \mu \mathrm{m}$; en $\mathrm{C}$ la escala $=3 \mu \mathrm{m}$; en $\mathrm{G}$ la escala $=5 \mu \mathrm{m}$. 
Torres-Colín et al.: Estudio palinológico de Macroptilium en México y Centroamérica
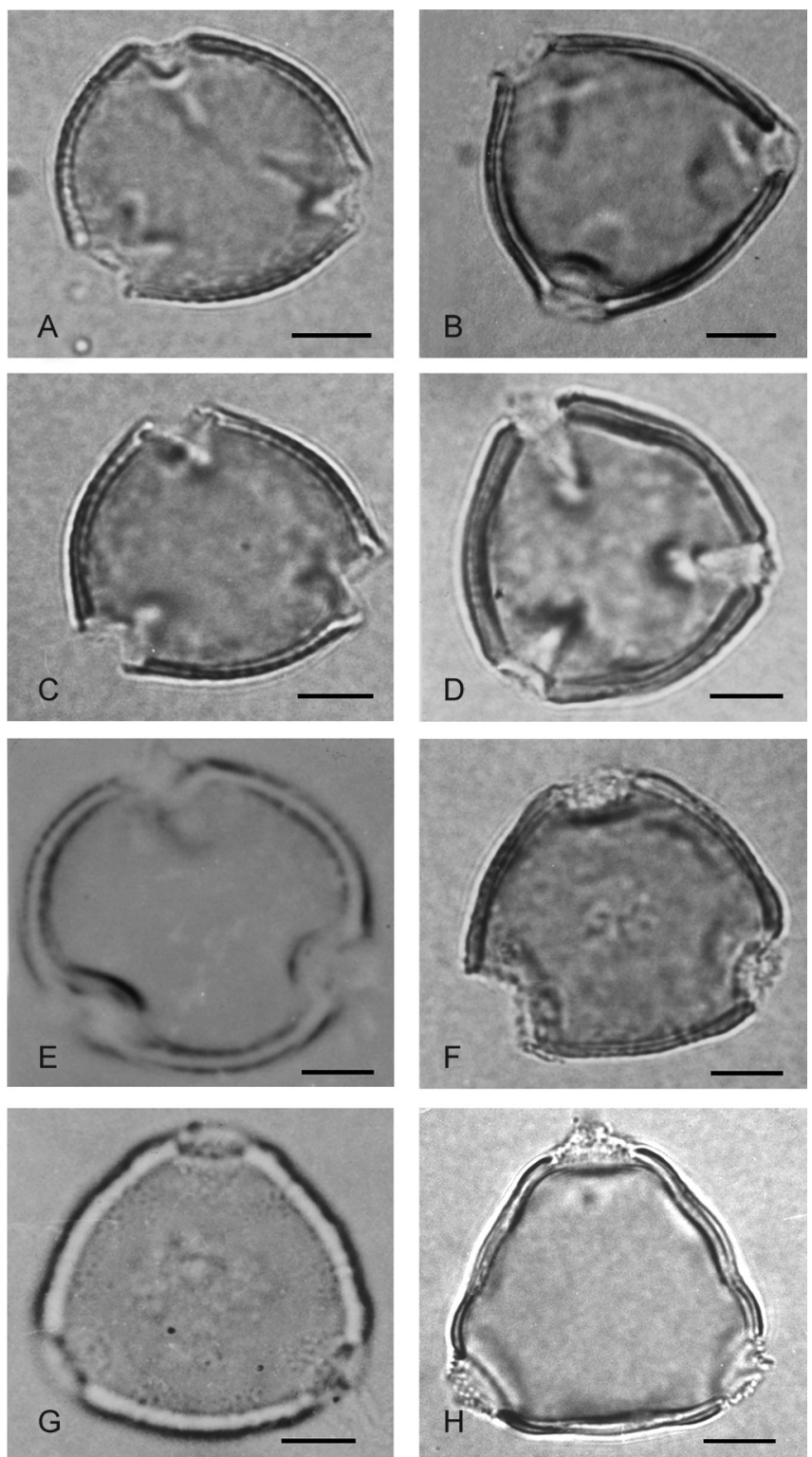

Fig. 2. Vista polar, granos de polen acetolizados de Macroptilium (microscopio óptico en campo claro). Se observa en D los colpos más largos y en A, B, C, E, F, G y H la diferencia en grosor de la exina (ver detalles de la membrana en fig. 3). A. M. atropurpureum. B. M. erythroloma. C. M. gracile. D. M. lathyroides. E. M. longipedunculatum (sección Macroptilium). F. M. gibbosifolium. G. M. pedatum. H. M. supinum (sección Microcochle). En A, C, E, F y H la escala $=4 \mu \mathrm{m}$; en B la escala $=6 \mu \mathrm{m}$; en D y G la escala $=5 \mu \mathrm{m}$. 

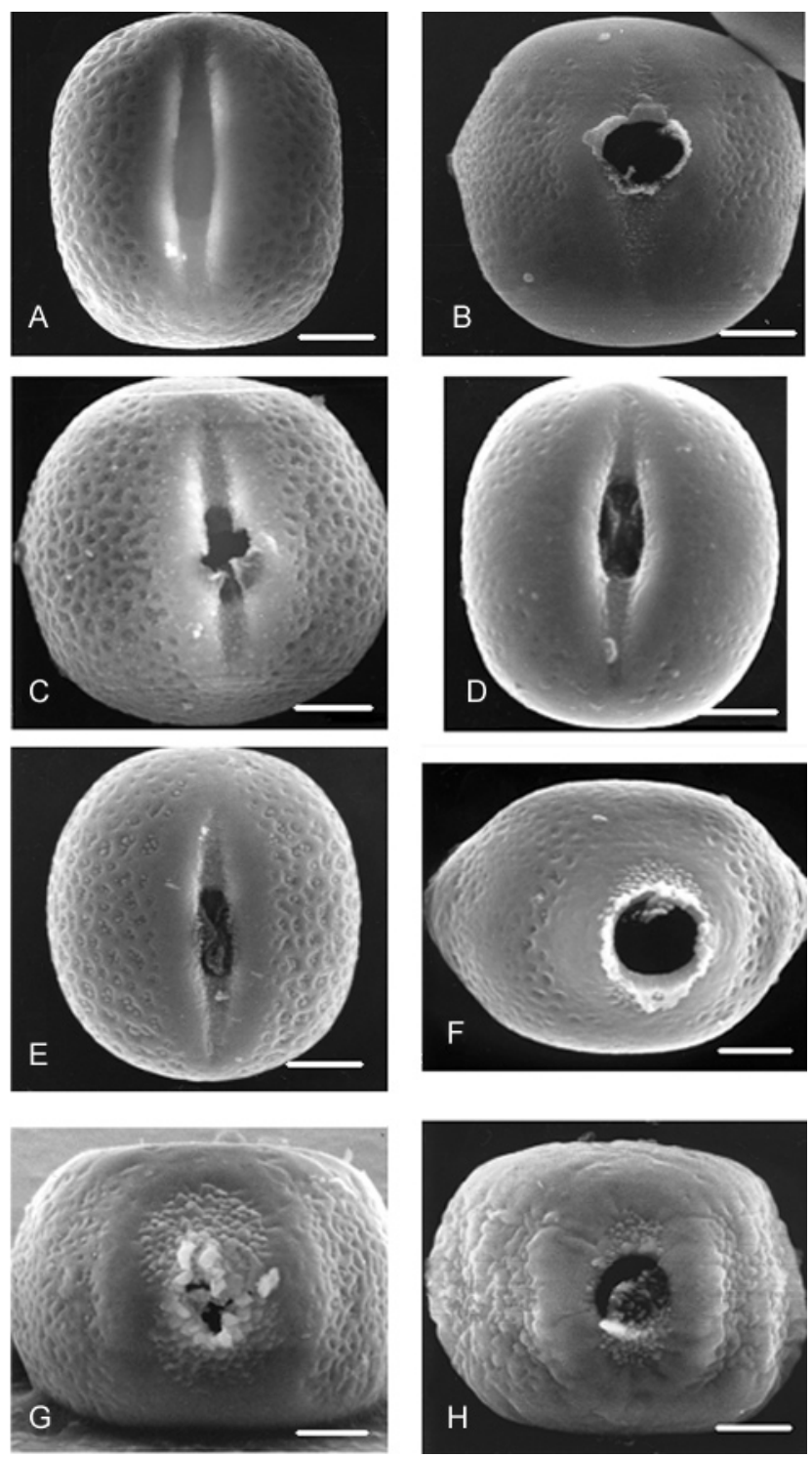

Fig. 3. Vista ecuatorial, granos de polen acetolizados de Macroptilium (MEB). Se observa margo no engrosado en A, B, C, D y E; margo engrosado, psilado en F y microrrugulado en $\mathrm{G}$ y H; membrana granular en A-H (ornamentación del mesocolpio detalle en fig. 5). A. M. atropurpureum. B. M. erythroloma. C. M. gracile. D. M. lathyroides. E. M. longipedunculatum (sección Macroptilium). F. M. gibbosifolium. G. M. supinum H. M. pedatum (sección Microcochle). En A la escala $=3.5 \mu \mathrm{m}$; en B, D, E y F la escala $=3.3 \mu \mathrm{m}$; en C y G la escala $=2.7 \mu \mathrm{m}$. 
Torres-Colín et al.: Estudio palinológico de Macroptilium en México y Centroamérica
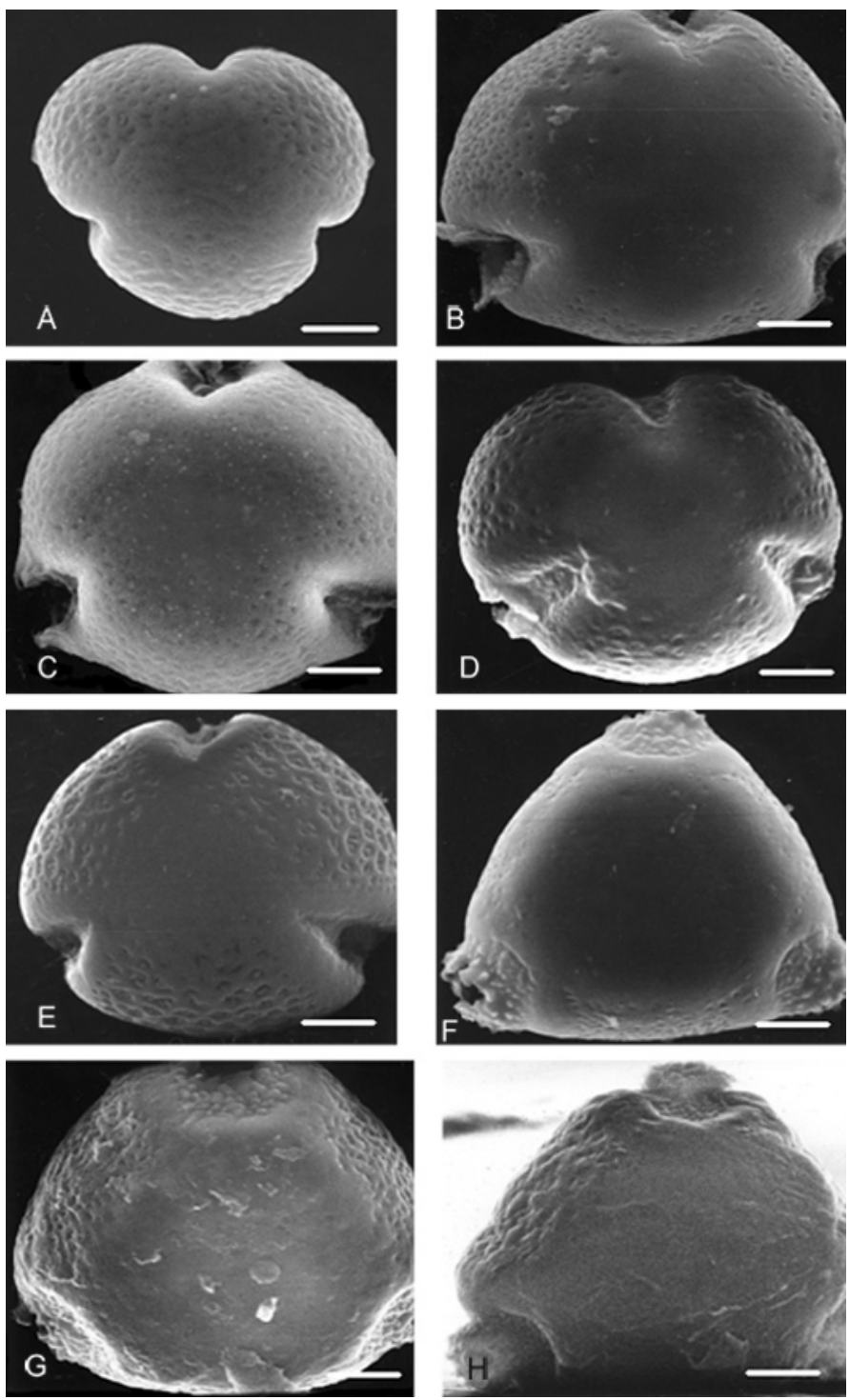

Fig. 4. Vista polar, granos de polen acetolizados de Macroptilium (MEB). Se observa contorno en vista polar, en A, C y E semiangular; en B y D circular; en F, G y H angular convexo; colpos largos en A, B, C, D y E; colpos cortos en F, G y H; ornamentación en apocolpio, en A, D, E, F y G punctado a foveolado; en B psilado; en C microrreticulado y en H rugulado. A. M. atropurpureum B. M. erythroloma. C. M. gracile. D. M. lathyroides. E. M. longipedunculatum (sección Macroptilium). F. M. gibbosifolium. G. M. supinum. H. M. pedatum (sección Microcochle). En A y F la escala = $4 \mu \mathrm{m}$; en B, C, D, E, G y H la escala $=$ $3 \mu \mathrm{m}$. 

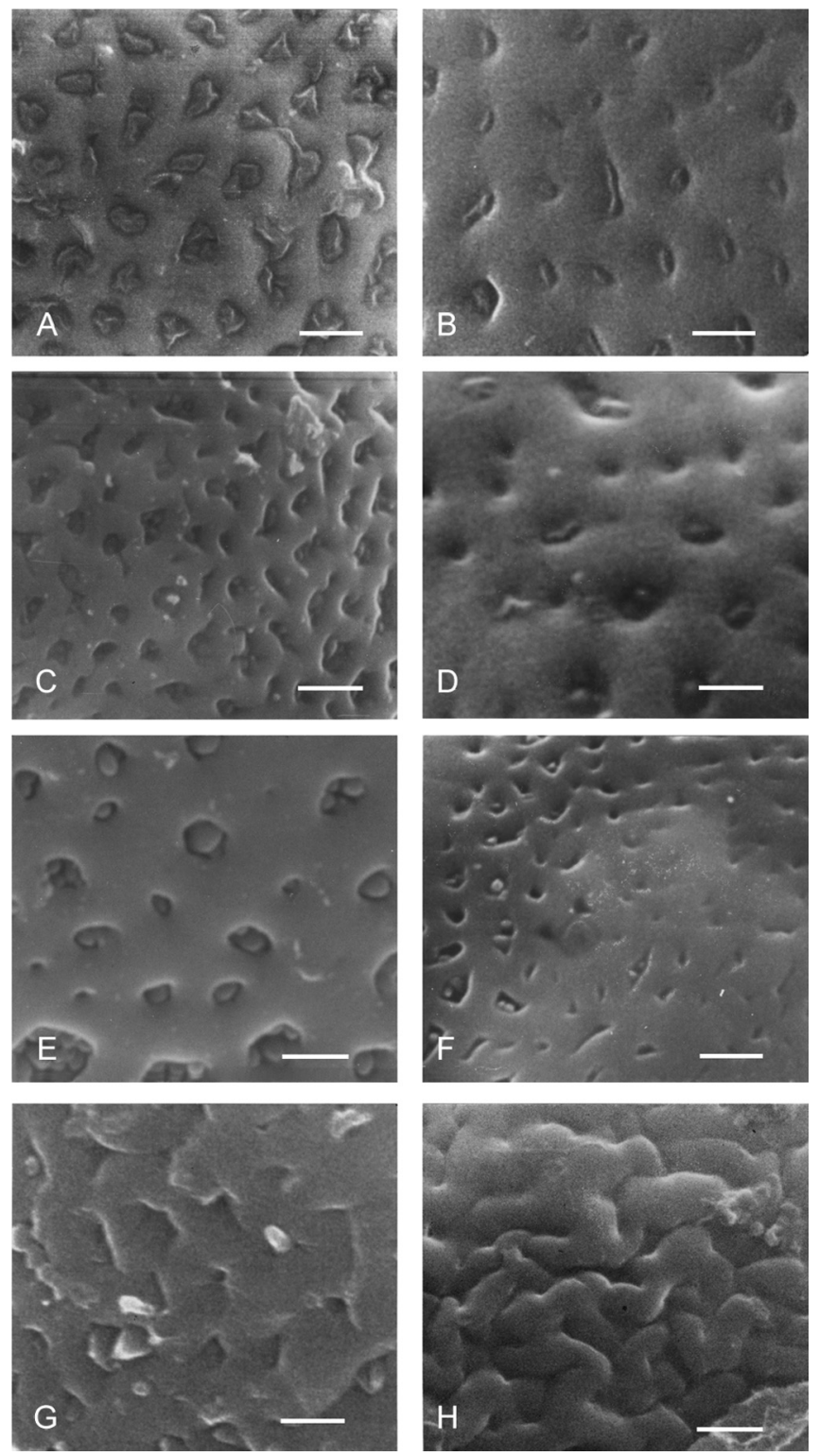

Fig. 5. Ornamentación del mesocolpio de Macroptilium (MEB). Se observa en A, B, C y E microrreticulado con lúmenes amplios; en D, F y G microrreticulado a foveolado con lúmenes pequeños; en H rugulado. Zonas con gránulos en todas las especies. A. M. atropurpureum. B. M. erythroloma. C. M. gracile. D. M. lathyroides. E. M. longipedunculatum (sección Macroptilium). F. M. gibbosifolium. G. M. supinum. H. M. pedatum (sección Microcochle). En A, C, F y H la escala $=1.8 \mu \mathrm{m}$; en B, D, E y G la escala $=1.2 \mu \mathrm{m}$. 
no creemos que tal clase de abertura se desarrolle en los granos de polen de género, dado el número de microsporas examinadas para cada taxon en este trabajo y las observaciones realizadas en las especies sudamericanas, de manera que lo que los mencionados autores reportan como triporado corresponde a tricolporado brevicolpado en el presente estudio.

Con relación a la longitud, claramente pudimos diferenciar a los granos del Tipo I caracterizados por brevicolpos, en $M$. gibbosifolium, M. pedatum y M. supinum (sección Microcochle, Figs. 3F-H; Cuadro 1) y a los del Tipo II por sus longicolpos, en $M$. erythroloma, M. lathyroides, M. gracile, M. atropurpureum y $M$. longipedunculatum (sección Macroptilium, Figs. 3A-E; Cuadro 1).

El margo o engrosamiento presente en los márgenes de la ectoabertura y los ángulos en el contorno en vista polar son caracteres más evidentes en las especies del Tipo I (sección Microcochle, Figs. 2F-H y 3F-H), en las cuales se observa un contorno polar angular convexo y un engrosamiento del margo de 2.4-3.5 $\mu \mathrm{m}$; en contraste con las del Tipo II (sección Macroptilium, Figs. 2A-E y 3A-E), donde el contorno es circular a semiangular y los margos varían de 1.0-2.8 $\mu \mathrm{m}$.

En la mayoría de las especies las endoaberturas se observaron lolongadas, con excepción de M. longipedunculatum que las presenta lalongadas (Fig. 3A-H). En la sección Macroptilium las endoaberturas tienen una longitud de casi $35 \%$ del colpo y en la sección Microcochle de 65\%.

La exina presenta una ornamentación particular en M. pedatum (Fig. $5 \mathrm{H}$ ), que la diferencia del resto de las especies del Tipo I, por desarrollar una exina semitectada rugulada muy homogénea tanto en el apocolpio como en el mesocolpio, contraria a la observada para M. gibbosifolium (Fig. 5F) y M. supinum (Fig. 5G), donde el apocolpio es tectado, punctado y el mesocolpio semitectado, microrreticulado. En el Tipo II, la ornamentación diferencia claramente a M. erythroloma de los demás componentes, por desarrollar una exina tectada y psilada en el apocolpio (Figs. 3B y 4B).

El grosor de la exina tiene un intervalo de 1.3-2.8 $\mu \mathrm{m}$, alcanzando el valor máximo en $M$. pedatum (Cuadro 1) pero con diferencias muy marcadas entre la sexina y la nexina. En el Tipo I, la sexina puede ser ligeramente más delgada que la nexina en $M$. gibbosifolium y $M$. supinum, o más gruesa que la nexina en $M$. pedatum. Para el Tipo II la sexina es del mismo grosor que la nexina en todas las especies, salvo en M. longipeduculatum.

Los dos tipos polínicos encontrados proporcionan elementos a la taxonomía de Macroptilium apoyando la existencia de las secciones anteriormente reconocidas por Drewes (1996) y Torres-Colín (2006), así como la subdivisión en dos secciones 
sugerida en la hipótesis filogenética propuesta por Espert et al. (2007), confirmando la inclusión de Macroptilium gibbosifolium, M. pedatum y M. supinum dentro de la sección Microcochle y la pertenencia de M. atropurpureum, M. erythroloma, M. gracile, M. lathyroides y M. longipedunculatum en la sección Macroptilium.

Para algunas especies los caracteres palinológicos aquí estudiados, resultaron taxonómicamente informativos en cuanto a su delimitación, tal es el caso de la exina más gruesa, rugulada en el apocolpio y mesocolpio encontrada en $M$. pedatum, cuyo reconocimiento como especie había sido fuertemente cuestionado por algunos autores, como McVaugh (1987). También el caso de M. longipedunculatum y M. gracile que han sido consideradas como sinónimos (Fevereiro, 1987); sin embargo el polen de $M$. longipedunculatum probó ser diferente del registrado para M. gracile.

\section{AGRADECIMIENTOS}

Nuestro agradecimiento a los revisores anónimos por sus sugerencias para mejorar este manuscrito y a las siguientes personas: Dr. Enrique Martínez Hernández por sus comentarios a las descripciones palinológicas; Dr. Mario Sousa Sánchez, jefe en turno del Herbario Nacional (MEXU), por permitirnos la toma de muestras para el estudio; M. en C. M. R. García Peña por la asistencia en el manejo de préstamos; D. G. Julio César Montero Rojas, por su valiosa ayuda con el escaneo y la elaboración de las figuras y a Felipe Villegas por el apoyo técnico en el montaje de las fotografías.

\section{LITERATURA CITADA}

Beyra, A. \& G. Reyes. 2005. El género Macroptilium (Benth.) Urb. (Leguminosae) en Cuba. Anales Jard. Bot. Madrid 62(2): 1-10.

Delgado-Salinas, A. \& L. Torres-Colín. 1995. Combinación nueva en el género Macroptilium (Fabaceae). Anales Inst. Biol. Univ. Nac. Autón. México, Bot. 66(2): 189-191.

Delgado-Salinas, A. \& L. Torres-Colín. 2004. New combinations in South American Phaseolinae: Dolichopsis and Macroptilium (Fabaceae: Phaseoleae). Novon 14: 424427.

Delgado-Salinas. A. \& G. P. Lewis. 2008. A new species of Macroptilium (Benth.) Urb. (Leguminosae: Papilionoideae: Phaseolinae) from North-Eastern Brazil. Kew Bull. 63: $151-154$. 
Drewes, S. I. \& R. A. Palacios. 1994. Nueva combinación en el género Macroptilium (Benth.) Urban (Leguminosae-Phaseoleae): Macroptilium psammodes (Lindman) S. I. Drewes \& R. A. Palacios. In: Ramella, L. \& P. Peret (eds.). Notulae ad Floram Paraquaiensem. Sér. Doc. Conserv. Jard. Bot. de Genève 49(1): 256-257.

Drewes, S. I. \& R. A. Palacios. 1995. Nota sobre la identificación de Macroptilium panduratum (Benth.) Maréchal y Baudet (Leguminosae-Phaseoleae), su presencia en Paraguay. In: Ramella, L. \& P. Peret (eds.). Notulae ad Floram Paraquaiensem. Sér. Doc. Conserv. Jard. Bot. de Genève 49(1): 540-542.

Drewes, S. I. 1996. Estudio palinológico de las especies argentinas de Macroptilium (Fabaceae). Darwiniana 34(1-4): 233-244.

Drewes, S. I. 1997. El género Macroptilium (Fabaceae) en la flora de Argentina. Bol. Soc. Argent. Bot. 32 (3-4): 195-216.

Drewes, S. I. 2001. Macroptilium (Benth.) Urban. In: Hunziker, A. T. (ed.). Flora Fanerogámica Argentina. Fascículo 75. Proflora-Conicet, Córdoba, Argentina. pp. 4-10.

Erdtman, G. 1952. Pollen morphology and plant taxonomy. Angiosperms. Almqvist and Wiksell. Stockholm. 539 pp.

Erdtman, G. 1960. The acetolysis method, a revised description. Svensk Bot. Tidskr. 54: 561-564.

Espert, S. M., S. I. Drewes \& A. D. Burghardt. 2007. Phylogeny of Macroptilium (Leguminosae): morphological, biochemical and molecular evidence. Cladistics 23: 119-129.

Fevereiro, V. P. B. 1986. Macroptilium (Bentham) Urban do Brasil (Leguminosae-FaboideaePhaseoleae-Phaseolinae). Arch. Jard. Bot. Rio de Janeiro 28: 109-180.

Juárez, F. \& S. Pérez. 1986. Nueva combinación en el género Macroptilium (Fabaceae). Kurtziana 18: 155.

Juárez, F. \& S. Pérez. 1987. El género Macroptilium (Fabaceae) en la provincia de Salta, Argentina. Anales INTA Salta 1: 31-42.

Maréchal, R. 1974. Deux combinaisons nouvelles dans le genre Macroptilium Urb. (Papilionaceae). Bull. Jard. Bot. Belg. 44: 443-444.

Maréchal, R. \& J. C. Baudet. 1977. Combinaisons nouvelles dans le genre Macroptilium Urb. (Papilionaceae). Bull. Jard. Bot. Belg. 47: 257-265.

Maréchal, R., J. M. Mascherpa \& F. Stainier. 1978a. Combinaisons et noms nouveaux dans les genres Phaseolus, Minkelersia, Macroptilium, Ramirezella et Vigna. Taxon 27(2/3): 199-202.

Maréchal, R., J. M. Mascherpa \& F. Stainier. 1978b. Étude taxonomique d'un groupe complexe d'espèces des genres Phaseolus et Vigna (Papilionaceae) sur la base de données morphologiques et polliniques, traitées par l'analyse informatique. Boissiera 28: $1-273$.

McVaugh, R. 1987. Leguminosae. In: Anderson, W. R. (ed.). Flora Novo-Galiciana. Vol. 5. The University of Michigan Press. Ann Arbor. 786 pp.

Melhem, T. S. 1971. Pollen grains of plants of the "Cerrado" Leguminosae-Lotoideae: tribe Phaseoleae. Hoehnea 1: 119-151.

Praglowski, J. \& W. Punt. 1973. An elucidation of the microreticulate structure of the exine. Grana Palynol. 13: 45-50. 
Punt, W., S. Blackmore, S. Nilsson \& A. Le Thomas. 1994. Glossary of pollen and spores terminology. LPP Contrib. Ser. No. 1. LPP Found., Utrecht. 71 pp.

Saxena, M. R. 1993. Palynology, a treatise. Oxford and IBH Publishing Co. PVT. LTD. New Delhi. 127 pp.

Stainier, F. 1974. Contribution à l'étude palynologique des Papilionaceae-PhaseoleaePhaseolinae III. Etude de quelques espèces des genres Phaseolus L., Vigna Savi et Physostigma Balf. Bull. Jard. Bot. Belg. 44: 1-15.

Taylor, A. S. 1966. Estudios sobre el polen de Phaseolus. Turrialba 16: 7-14.

Torres-Colín, M. L. 2006. Revisión taxonómica del género Macroptilium (Benth.) Urb. (Leguminosae) en México y Centroamérica. Tesis de maestría (Maestría en Ciencias, Biología). Facultad de Ciencias, Universidad Nacional Autónoma de México. México, D.F. 171 pp.

Urban, I. 1928. Leguminosae. In: Plantae cubenses novae vel rariores. L. Ekman lectae. IV. Symb. Antill. 9(4): 433-543.

Verdcourt, B. 1970. Studies in the Leguminosae-Papilionoideae for the flora of tropical East Africa. IV. Kew Bull. 24: 507-525. 


\section{APÉNDICE}

Material examinado de Macroptilium

Macroptilium atropurpureum (Sessé \& Moc. ex DC.) Urb. GUATEMALA. Clausen 6127 (MEXU). MÉXICO. Baja California, Luckow 2828 (MEXU); Estado de México, Hinton 2269 (MEXU); Jalisco, Torres Colín 1070 (MEXU); Oaxaca, Lorence 4825 (MEXU). NICARAGUA. Sousa Sánchez 13003 (MEXU).

Macroptilium erythroloma (Mart. ex Benth.) Urb. NICARAGUA. Stevens 10205 (MEXU). PERÚ. Delgado 2080 (MEXU).

Macroptilium gibbosifolium (Ortega) A. Delgado. GUATEMALA. Véliz 14137 (MEXU). HONDURAS. Chorley 138 (MEXU). MÉXICO. Distrito Federal, Torres Colín s.n. (MEXU); Guerrero, Delgado 1079 (MEXU); Jalisco, Delgado 245 (MEXU); Oaxaca, Cedillo 832 (MEXU); Sonora, Gentry 1708 (MEXU).

Macroptilium gracile (Poepp. ex Benth.) Urb. BELICE. Whitefoord 2713 (MEXU). MÉXICO. Chiapas, Breedlove 54459 (MEXU); Sousa Sánchez 12253 (MEXU); Oaxaca, Cedillo 717 (MEXU); Torres 85 (MEXU). NICARAGUA. Sousa Sánchez 12918 (MEXU).

Macroptilium lathyroides (L.) Urb. BELICE. Dwyer 14696 (MEXU); Whitefoord 2190 (MEXU). MÉXICO. Chiapas, Ramos s.n. (MEXU); Tabasco, Cabrera 5314 (MEXU); Veracruz, Ventura 11862 (MEXU). NICARAGUA. Stevens 22452 (MEXU).

Macroptilium longipedunculatum (Mart. ex Benth.) Urb. MÉXICO. Guerrero, Ladd 262 (MEXU); Jalisco, Delgado s.n. (MEXU); Torres Colín 1073 (MEXU); Michoacán, Soto Núñez 3561 (MEXU); Oaxaca, Sousa Sánchez 6383 (MEXU); Sousa Sánchez 8214 (MEXU). PANAMÁ. Knapp 3272 (MEXU).

Macroptilium pedatum (Rose) Maréchal \& Baudet. MÉXICO. Guerrero, Pringle 8367 (MEXU); Soto Núñez 1777 b (MEXU); Michoacán, Soto Núñez 3555 (MEXU).

Macroptilium supinum (Wiggins \& Rollins) A. Delgado \& L. Torres-Colín. MÉXICO. Nayarit, Delgado 1045 (MEXU); Torres Colín 1061 (MEXU). 